\title{
Membrane transport and flux response
}

\author{
Masahisa 'Mabo' Suzuki
}

The Danish physiologist Ussing was the first to present the use of the flux-ratio as an investigative tool in membrane transport studies $[3,4]$. In a set of two complementary experiments, steady unidirectional outfluxes of a tracer from opposite faces of a membrane slab are measured. In the first experiment, face $A$ of the slab is kept at constant tracer concentration, while face $B$ is washed at zero concentration, and the steady outflux $j_{\text {out }}$ at face $B$ is measured. In the second experiment, the roles of faces $A$ and $B$ are interchanged, and the steady outflux $j_{\text {out }}^{*}$ at face $A$ is measured.

Ussing showed that if the membrane is transversely homogeneous, the ratio of the first outflux to the second in the reverse direction is proportional to the ratio of the input source concentrations, and gave the constant of proportionality in terms of the diffusion coefficient and the convective (drift) velocity, assuming that these could depend arbitrarily on distance into the slab from one face, so that tracer transport, although linear, is not in general symmetric between the two experiments. Well-known as "Ussing's flux-ratio theorem" [4], the result spawned many generalisations and extensions in relation to variable physical properties in the membrane and more general boundary conditions, and, most remarkably, was extended to unsteady situations $[\mathbf{1}, \mathbf{2}]$. However, several questions are not yet fully answered in this area of study: "What does outflux reciprocity (constancy of the outflux-ratio at all times) or its failure tell us about membrane transport?" "What can be said about behaviour at the inflow boundaries?" "What else other aspects of membrane transport can Ussing-type input-output experiments reveal?"

In this project, mathematical analyses were carried out on flux responses in several model systems of membrane transport, in order to seek answers to these questions. The models of investigation were based on Ussing-type experiments in order to try and make possible a kind of 'black-box' input-output analysis of membrane transport systems.

A new flux relationship is found in which a constant difference between complementary unsteady influxes $j_{\text {in }}$ and $j_{i n}^{*}$ is maintained in a linear transport system with spaceindependent diffusion and convection. This result is equally as simple as an Ussing-type

Received 7th May, 1997.

Thesis submitted to the University of Queensland June 1996. Degree approved 15th November, 1997. Supervisor: Professor AJ Bracken.

Copyright Clearance Centre, Inc. Serial-fee code: 0004-9729/97 \$A2.00+0.00. 
outflux-ratio relationship, and should provide an important test for longtitudinal heterogeneity in membrane transport, just as the outflux-ratio provides a test for transverse heterogeneity [4].

However, both flux relationships, Ussing's outflux ratio, and the newly found influx difference, fail to hold over time in an general asymmetric nonlinear transport system, while it is obviously true that both relationships must be maintained for all time if the geometry of the experiments is symmetric. The early-time study shows that the influxdifference is spatially dominated, and the inflow relationship is valid only if diffusionconvection parameters are space-independent. On the other hand, the outflux-ratio is found to differ from the value as in a linear transport system. These early-time studies indicate no further generalization of flux theorems other than the results obtained in this project for new influx relationships, or in the past for outflux relationships.

To describe somewhat different behaviours of transport and flux responses from studies in the past, a new graphical investigative tool is presented to identify one particular infinite-time property of a general transport system. It involves a simple experimental procedure without the need to obtain explicit solutions of transport equations in general, using a special 'time-lag' asymptotically derived from accumulated fluxes. Since this investigative tool is expressed in terms of accumulated fluxes, which have the same dimensions (units) as a concentration of a substance, this application should require technically simple concentration measurements in practice, as opposed to measuring real fluxes. As an application of this investigative tool, a general expression for chemical content in a domain is obtained for a general nonlinear transport system in terms of the 'time-lag' and accumulated fluxes for inflow and outflow boundaries. This investigative tool is also used to examine Ussing-type reciprocity properties for general nonlinear transport systems in complementary experiments. It should have application to a general linear/nonlinear transport system in practice because of its simplicity.

In another application of Ussing-type theorems, a linear transport system is derived from a nonlinear transport system, in order to obtain a constant ratio for Ussing-type complementary outfluxes, involving experiments starting from a state of chemical equilibrium. These experiments were originally designed to trace radio-active markers in a biological or physiological system. Although the nonlinear transport model used in this study is a second order nonlinear system, the general matrix form obtained should allow application of the concept to a general nonlinear transport system, working from a state of chemical equilibrium.

The models considered in this project were intended to be fundamental and, as far as possible, to represent a wide class of mathematical models of transport systems. The results should be useful experimentally to test the validity of models of membrane transport and flux response. 


\section{REFERENCES}

[1] L. Bass and A.J. Bracken, 'The flux-ratio equation under nonstationary boundary conditions', Math. Biosci. 66 (1983), 87-92.

[2] O. Sten-Knudsen and H.H. Ussing, 'The flux ratio equation under nonstationary conditions', J. Membrane Biology 63 (1981), 233-242.

[3] H.H. Ussing, 'The distinction by means of tracers between active transport and diffusion', Acta Phys. Scand. 19 (1949), 43-56.

[4] H.H. Ussing, 'Interpretation of tracer fluxes', in Membrane transport in biology, (G. Giebisch, D.C. Tosteson and H.H. Ussing, Editors) 1 (Springer-Verlag, Berlin, Heidelberg, New York, 1978), pp. 15-40.

Department of Mathematics

The University of Queensland

Queensland 4072

Australia 